\title{
El tratamiento multiestratégico en la investigación sociológica
}

\author{
Carlos Lozares \\ Antonio M artín \\ Pedro López
}

Universitat Autònoma de Barcelona. D epartament de Sociologia

08193 Bellaterra (Barcelona). Spain

\section{Resumen}

Este artículo pretende aportar una reflexión metodológica en una dirección que, siguiendo a D erek Layder (1993) denominamos «aproximación multiestratégica». Esta orientación propone un modelo y un proceso metodológico que trata de captar y analizar el hecho social investigado como una totalidad a partir de las distintas pertinencias que lo componen, sin que sea reducido o segmentado en su identidad por los efectos de modelo y método de análisis utilizado. Esta metodología es la que ha sido seguida, en líneas generales, en esta investigación como la más apropiada para el tratamiento de una realidad social que, como la estudiada, está atravesada por dimensiones múltiples y contiene un elevado grado de complejidad. Se da cuenta de sus objetivos, de la comparación con otras metodologías, de los elementos que la componen así como de la correspondencia y adecuación entre la metodología de nuestra investigación y dicha perspectiva de análisis.

Este artículo es parte de una investigación cuyos resultados aparecen en este mismo número de PAPERS. El artículo tiene autonomía y entidad por sí mismo pero la lectura del resto de los artículos de este número facilitará su contextualización.

Palabras clave: metodología, diseño de investigación, métodos cuantitativos y cualitativos.

\section{Abstract}

This article seeks to contribute a methodological reflection in a direction that, following D erek Layder (1993) we call the M ulti-strategy Approach. This approach proposes a model and a methodological process that aims to grasp and analyse the social phenomenon as a totality through its different aspects without its identity being reduced or segmented by the effects of the model and the method of analysis. This methodology was generally followed in this research project as being the most appropriate for dealing with a social real ity composed of interlinking dimensions and involving a high degree of complexity. We present the objectives, make a comparison with other methods, and study the elements of which it is composed and the correspondence and adaptation of our research methodology to this perspective of analysis.

This paper is part of a research work whose results are in this PAPERS volume. The paper is autonomous but the reading of the other papers of this volum can help to contextualize it.

Key words: methodology, design of research, quantitative and qualitative methods. 


\section{Sumario}

0 . Introducción

1. La investigación multiestratégica

2. La investigación realizada descripción y objetivo
3. La aproximación multiestratégica de nuestra investigación

Bibliografía

\section{Introducción}

Los modelos y métodos de investigación en sociología se presentan, a veces, excesivamente salomónicos. Las alternativas, al menos en los manuales de investigación, parecen dirigirse, o bien únicamente hacia los aspectos y contenidos macrosociales de los fenómenos, o bien se inclinan solamente hacia los micros con sus métodos correspondientes. El enfoque multiestratégico propone, a partir de la naturaleza articulada del hecho social, tener presente en la investigación los diferentes planos/dimensiones de los que se compone todo fenómeno social: la acción y la situación en sus aspectos más individuales e interaccionales y los contextos sociales estructurales e institucionales más amplios, además de tener en cuenta también las vinculaciones mutuas que se dan entre estos niveles. Frente a una perspectiva de investigación en la que la explicación buscada, o bien se orienta a validar teorías (hipótesis) previamente elaboradas o bien se orienta solamente a explorar, crear o generar nuevos conceptos y teorías, el enfoque multiestratégico, en un intento de acercarse más a la práctica real de la investigación sociológica, propone puentes y vías de acceso entre ambos tipos de explicación/comprensión. Por fin, frente a la utilización, o bien de modelos, procedimientos y técnicas exclusivamente experimentales, métricos, estadísticos y, en general, cuantitativos o bien exclusivamente etnográficos, esta línea de investigación propone la aplicación complementaria de dichos métodos en los diferentes planos de objeto estudiado y en las distintas fases de la investigación.

En las últimas décadas, en particular la de los ochenta y con más intensidad en lo que va de la actual, ha existido una gran preocupación por esta polarización y poco a poco se va dando una reflexión teórica y una investigación empírica en la dirección de encontrar síntesis entre las perspectivas micro y macro; entre aquéllas que se centran más en la estructura social y las que se inclinan más hacia el análisis de la (inter)acción social. Se es consciente de que la disyunción de estas polaridades representa uno de los problemas más espinosos y una de las tareas más urgentes del quehacer en sociología. Aunque esta preocupación estuvo también presente en los clásicos, lo que ha caracterizado a la sociología, sobre todo a partir de la segunda mitad del siglo, es el divorcio entre dichos enfoques. Las manifestaciones son múltiples: por un lado, las formas estructuralistas de diversa índole, el funcionalismo a secas o el estructural, el determinismo cultural o normativo; por otro, las corrientes fenomenológicas, interaccionistas, etnometodológicas, etc. por hablar de las relevantes. Como decíamos, el interés comienza a centrarse en teorías y prácticas de investiga- 
ción que conduzcan a una mayor integración y superación de la alternativa macro-microsociología.

Estos intentos, como indica G. Ritzer (1992) van en varias direcciones: la teoría de la elección racional, en concreto, J. Coleman; R. Collins en los análisis rituales interaccionales; los análisis conversacionales de A. Cicourel y K norrCetina; la teoría del intercambio de Emerson; algunos análisis macro con base freudiana; el enfoque multidimensional de J. Alexander entre orden colectivo-individual y acción instrumental-normativa; la teoría integradora de las redes de R. Burt en su intento de salvar el abismo entre orientaciones atomistas y normativas; los modelos de R. Ritzer de construcción de interacción dialéctica entre los niveles macro y microsociológicos, entre los planos objetivos y subjetivos; las teorías del interactor de J. Berger, D .P. Eyre, M . Zelditch que se centran en los actores y en sus interacciones.

La dificultad de encontrar teorías y niveles de análisis sintéticos tropieza con obstáculos añadidos, como es el de llegar a definiciones homogéneas de lo que se entiende por macro y micro, ya que puede darse la inclinación a reducir lo micro a lo psicológico y lo macro al análisis sociodemográfico de las poblaciones.

D e hecho, al avanzar en esta dirección, se busca por ahora aliviar o amortiguar, aunque no resolver definitivamente, el dilema o quizás sólo disyuntiva, en el que parece está sumida y dividida la investigación sociológica. El camino en esta perspectiva consiste en contemplar y tratar el hecho analizado en su máxima totalidad al elaborar y aplicar una estrategia de investigación extraída de la identidad del propio objeto analizado y no de apriorismos metodológicos. Trata así de superar una metodología que considera (y aplica) un solo procedimiento como «verdadero» y al mismo tiempo se sitúa lejos del eclecticismo que proclama que todo vale para investigar. En este sentido no es el pluralismo metodológico lo que propugna, sino la indivisibilidad del hecho social y la multiplicidad de las pertinencias sociológicas que lo constituyen.

Lo que pretendemos con esta exposición es muy simple: presentar un caso de investigación que se acopla a este enfoque. No se trata, por consiguiente, de validar una perspectiva metodológica de la que solamente se dan por ahora unos apuntes o un esbozo, sino de ofrecer un trabajo que abunde en su enriquecimiento. Para una mejor comprensión de estos propósitos trataremos suce siva y sucintamente, primero, de lo que se entiende por aproximación multiestratégica en vista a una clarificación y fijación conceptual necesaria y, segundo, de dar cuenta, a partir de fases, planos y contenidos de análisis, de una investigación precisa como posibilidad de una tal orientación.

\section{La investigación multiestratégica}

En este apartado seguiremos la propuesta de D. Layder (1993), tratando de resumir sus puntos centrales en sucesivos epígrafes.

1. La aproximación multiestratégica se sitúa, como objetivo metodológico, entre dos perspectivas claramente diferenciadas en la investigación sociológica. 
Ambas líneas metodológicas dejan de lado, o no dedican la atención suficiente, a una serie de problemas o hechos que esta orientación procura abordar. Se trata, entre otros, de la relación entre la visión y el tratamiento macro y microsociales como, por ejemplo, la vinculación de los análisis institucionales y estructurales con los de la interacción social, de las situaciones, representaciones o imaginario simbólico de los individuos, etc. La propuesta intenta abrir un tipo de proceso entre ambas polaridades (D. Layder, 1993: 197-201).

2. M ás concretamente, D. Layder concibe y define la aproximación multiestratégica con relación a dos precisas orientaciones metodológicas, la teoría de rango medio y la teoría fundamentada (G rounded Theory), que, aunque se presentan como líneas clásicas de investigación en los manuales, su esquematismo puede dejar de lado otras estrategias potencial mente ricas para una explicación relevante y válida del hecho social. No entraremos en los elementos descriptivos de ambas perspectivas. $\mathrm{N}$ os remitimos al mismo Layder (1993: 2-5, 109-115) y a los autores citados por él mismo: por la teoría de rango medio a J. Goldthorpe (1980) y E. Wright (1980); y por la teoría de base fundamentada a B. Glaser y A. Strauss (1967), E. Becker (1970), L. Lofland (1971) y A. Strauss (1987).

3. Se ha de reconocer que, aunque la línea divisoria entre las perspectivas enunciadas parece clara, no siempre es así en las investigaciones concretas de sociología. Tampoco aparece suficientemente claro para el sociólogo investigador que concretamente ha de elaborar el objeto de estudio tenga que optar a priori exclusiva y necesariamente por una $\mathrm{u}$ otra de ambas orientaciones. $\mathrm{H}$ ay a $\mathrm{lo}$ largo de la historia de la investigación en sociología ejemplos suficientes de estudios difíciles de situar en uno u otro lado de tal barrera. Además, aunque parece claro que los métodos ligados a la teorías de rango medio son los que más se utilizan para validar hipótesis previamente enunciadas, sin embargo, se dan ocasiones en que también se utilizan para generar o construir conceptos o teorías en una dirección claramente exploratoria o de inicial tipificación. Las fronteras no aparecen tan netas (D. Layder, 1993: 3-4). Por lo demás es un hecho constatable, en la experiencia investigadora, que después de la realización de una investigación el sociólgo acostumbra a sentirse insatisfecho, si se limita exclusivamente a una de esta orientaciones, como si algo se le hubiera escapado del fluido e inaprensible hecho social.

4. D . Layder presenta un camino al ternativo entre ambas perspectivas en una aproximación realista o multiestratégica. N o se trata de una cuestión puramente nominal ni de generar procesos ambivalentes 0 ambiguos de investigación. D. Layder la define como una orientación de investigación que construye el objeto de estudio a partir de «múltiples cortes analíticos o metodológicos para llevar a cabo una cobertura empírica y teóricamente densa del área investigada». La naturaleza nivelada, o la posibilidad de establecer cortes analíticos, en los objetos sociales, la consideración de que la actividad social no puede 
analizarse fuera del contexto espacial y temporal y, por fin, la integración de los rasgos macro y micro en la vida social (D. Layder, 1993: 107-109), comportan una correspondiente metodología de investigación. Son pues fundamentales los diversos cortes analíticos que se producen en los objetos estudiados y la consiguiente apertura estratégica en la recogida y el tratamiento de los datos: métodos y estrategias que han de permitir una gran flexibilidad para maximizar la generación de teoría y, más concretamente, la seguridad de que «son usados directamente al servicio de la comprensión de los vínculos que ligan el mundo interpersonal de la vida de cada día con el mundo más impersonal de las instituciones sociales» (D . Layder, 1993: 205-206).

5. Esta visión no impone a priori una teoría rígida en la investigación, sino que facilita su emergencia. Pero hay que añadir que la investigación ha de hacerse en un contexto de supuestos generales, lo más especificados posibles, sobre la naturaleza de los vínculos entre los aspectos macro y micro de la realidad social analizada y sobre los contenidos y las relaciones entre los diversos niveles a que dan lugar los cortes analíticos. No hay rigidez inicial de modelo a validar. Sin embargo, ha de ser clara la construcción y el contenido de los distintos niveles del hecho social y su vinculación. N o se trata, pues, de un inductivismo simple o estrecho, ya que, de ninguna manera, los hechos hablan por sí mismos si no los hacemos hablar. Para D. Layder las más apropiadas y productivas estrategias de investigación son aquéllas que incorporan esta visión que ha de ser también una forma de disciplina investigadora controlada. Ello implica multiplicidad de métodos y flexibilidad de diseño para la cobertura teórica y empírica.

6. Comenzando por la naturaleza nivelada, D. Rayder construye un «mapa» que da cuenta, al mismo tiempo, de los niveles del hecho social y de la consiguiente integración de los mismos: el "sí mismo», el de la «actividad situada», el del «marco o composición», el del «contexto», el de la «historia» y el del «poder». Son diferentes planos analíticos de un mapa que reconstruye y compone el hecho social analizado (D . Layder, 1993: 205). Establece potenciales áreas de investigación que tienen también como objetivo el orientar y sugerir interrogantes antes y durante el proceso de investigación. Los procedimientos de análisis han de corresponder a ellos si se desea dar cuenta de la diversidad y de la plenitud del hecho social. Veamos el contenido escueto de los cuatro primeros niveles.

D entro del «sí mismo» se incluyen las relaciones que el individuo mantie ne con el entorno social, así como la interiorización socializada de las mis mas: se trata de la intersección de la experiencia biográfica y dicho entorno. Sobre la «actividad situada» se focalizan los aspectos dinámicos emergentes de la interacción social. El «marco o composición» comprende las formas intermedias de organización social como escuelas, hospitales, fábricas, etc. El «contexto» se refiere al marco general macrosocial como forma que proporciona el más amplio entorno de la actividad social: género, categoría social, 
edad, etc. No hay claros y estrictos límites entre ellos, pero tienen elementos suficientes para atribuirles contenidos consistentes. A demás de estos niveles que constituyen la dimensión vertical o sincrónica del mapa, D . Layder introduce la dimensión horizontal temporal que opera y da los aspectos dinámicos y diacrónicos. La escala temporal es fundamental: si la «congelamos» en un momento tendremos una visión sincrónica y un tanto deformada de la realidad, ya que la actividad social es un proceso. Sin perder esta visión global, horizontal y vertical, del hecho social, el interés del sociólogo investigador puede centrarse más en una dimensión que en otra, en un nivel que en otro (D. Layder, 1993: 8-11).

7. Veamos al gunas otras consideraciones sobre la investigación de aproximación multiestratégica. La investigación AM S necesita ser sistemática y flexible. Sistemática, porque ha de maximizar la posibilidad de emerger y generar teoría sin dejarlo a la aleatoriedad del descubrimiento casual. Flexible, porque ha de ser capaz de responder a problemas no anticipados. La flexibilidad permitirá organizar y reformular el diseño inicial a la luz de los problemas y soluciones inesperados (D ouglas, 1976; Glaser y Strauss, 1967; Layder, 1993: 121).

Aunque el objetivo principal de esta perspectiva no es precisamente la validación y generalización de hipótesis teóricas, no está excluido que haya aspectos parciales de la investigación que cumplan dicho objetivo y sobre todo que la combinación de tipos de datos y métodos permita confirmar por un procedimiento las exploraciones o hipótesis derivadas de otro método (D . Layder, 1993: 121-122).

Este procedimiento tiende a estimular la máxima utilización de datos en todos los niveles de la investigación, cubriendo la mayor área posible del objeto (D. Layder, 1993: 122-124).

8. Por fin, veamos al gunas líneas o campos de más interés para la aplicación de esta metodología.

Esta orientación metodológica será evidentemente más útil y eficaz allí donde el investigador se propone realizar, en el objeto analizado, diversos cortes analíticos y metodológicos siempre que la naturaleza del objeto y la teoría implícita lo permitan. Es de particular interés allí donde el objeto de análisis consiste precisamente en la vinculación entre aspectos macro y micro de la vida social.

Será también adecuada para identificar y/o estructurar los conceptos. A partir de un concepto inicial sugerente, incluso impreciso, se puede llegar a una organización provisional de los datos y sugerir otras ideas o conceptos más elaborados que sean más aproximados al objeto. Es particularmente adecuada cuando se albergan, dentro de los contenidos y conceptos, rasgos objetivos y subjetivos implícitos o explícitos.

Es apropiada en el análisis comparativo de situaciones de interacción entre individuos, entre grupos o entre individuos y grupos, siempre que se inscriban en contextos más amplios, por ejemplo, la interacción en un bar-café com- 
parada con las interacciones en los transportes públicos, las relaciones entre dependiente y cliente en el mercado comparadas con las de estudiante y profesor en una universidad.

Se puede también ir más lejos elaborando y desarrollando tipologías o modelos que clasifican o dan cuenta de tipos de grupos, situaciones o relaciones más amplias. Estas tipologías se pueden referir a infinidad de formas institucionales tales como prisiones, asilos, mercado laboral, organizaciones políticas y religiosas, etc. Se pueden hacer análisis más sistemáticos indicando la caracterización de los fenómenos comparables, sus altas similitudes 0 diferencias existentes.

Trabajando con tipologías se puede llegar a construir muestras más sistemáticas y efectivas. Es la noción de G laser y Strauss del muestreo teórico y comparativo. Las muestras pasan a ser un hecho decisivo en la investigación. Los individuos o los grupos de gente que provienen de dichas muestras, establecidas sobre criterios comparativos, teóricos u observacionales, poseen la relevancia suficiente para una posible generalización, corroboración y, en su caso, una mejor validación de la intuida teoría inicial. Por otro lado, la introducción y el manejo en el proceso investigador de tipologías estructurales tiene la ventaja de añadir un poder nuevo en el camino de la validación (D. Layder, 1993: 206-207).

Por fin, dentro del dominio de esta aproximación, pueden al bergarse diversas formas de interacción entre métodos y técnicas si el objeto de análisis lo requiere. Así, el uso de métodos y datos cualitativos y en general etnográficos puede ser imprescindible para comprender y dar cuenta de las actividades e interacciones de los actores, así como de su significación, en diversas situaciones, por ejemplo, la de un taller, la vida social en una calle determinada, etc. Pero también es imprescindible el uso de métodos cuantitativos para contextualizar dichas situaciones en un marco más estructural y/o institucional. Se puede incluso recurrir a formas sofisticadas de cuantificación como un tipo de control de las interpretaciones de los datos cual itativos. Así, los datos cuantitativos pueden servir tanto para generar teoría en particular sobre los macroprocesos en los que se coloca la actividad situada, como para validar teoría, como es el caso habitual (D . Layder, 1993: 127-128).

\section{La investigación realizada: descripción y objetivos}

El objetivo de este apartado no consiste, como ya se señalaba al principio, en validar la concepción metodológica expuesta; tampoco la de presentar una construcción acabada y consistente de la misma. Este procedimiento no tiene, por ahora, una elaboración metodológica suficiente como para pretenderlo. Buscamos solamente mostrar cómo, a partir de una investigación precisa, se pueden desarrollar algunas concreciones de la misma y aplicar su lógica (para otras investigaciones que van en esta dirección, ver C. Lozares, y otros, 1988; C. Lozares, 1991). D ado que una explicación más detallada y global del contenido de la investigación se encuentra en el artículo precedente, damos cuenta brevemente de la investigación real izada insistiendo solamente en aquellos 
aspectos globales o parciales (contenido, objetivos, niveles y fases) que son más pertinentes a la aproxiación multiestratégica. Con ello podemos retener aquellos elementos que posibiliten una mejor comprensión de la tercera parte en la que retomamos los temas claves que relacionan nuestra investigación con la aproximación propuesta.

Para comprender mejor y más rápidamente lo esencial de la investigación realizada comenzamos, primero, por dar cuenta de su proceso dentro de los distintos niveles y fases. Pasamos después a explicar sucintamente sus objetivos básicos.

\subsection{Proceso de la investigación: fases y niveles de análisis}

\subsubsection{El primer nivel de análisis}

Tomando como base los datos originales de una gran encuesta representativa sobre las condiciones y hábitos de vida en la región metropolitana de Barcelona ${ }^{1}$, hemos construido diversas tipologías sobre tres ámbitos de la vida social, el trabajo productivo (TP), la dedicación al trabajo reproductivo (TR), y la disponibilidad en el tiempo libre (TL). Retomando estas tres tipologías, hemos confeccionado una tipología estructural que da cuenta de la relación entre dichos ámbitos. Esta tipología resultante es estrictamente cuantitativa y, debido a la articulación de ámbitos diferentes, la hemos denominado, dentro de nuestro modelo general de análisis, «tipología de articulación de la vida cotidiana». Para una mejor caracterización social de esta tipología se han introducido, en su identificación, factores sociales, como categorías sociales, sexo, edad, etc. Por ello no se trata sólo de una tipología de ámbitos diferenciados, sino que es también, en cuanto tal y por las validaciones realizadas, un buen descriptor social general. Tiene una función de orientación de la investigación; es de base estructural y contextual en los consecutivos análisis; es materia prima para la validación de una teoría de la segmentación y reproducción social y, por fin, es un mecanismo o instrumento de tipología teórica y comparativa.

\subsubsection{El segundo nivel de análisis}

Sobre la base de esta tipología construida se ha escogido una muestra cualificada de 200 individuos pertenecientes a cada uno de sus tipos. Se ha estudiado en los individuos que pertenecen a dichos tipos las representaciones y las actitudes centradas en cada uno de los ámbitos, TP, TR y TL. Se pretende, primero, profundizar más en la identidad de cada uno de los tipos a partir de contenidos diferentes que los definen como tipos de base y, segundo, comprobar la consistencia y coherencia, en este nivel de análisis, de los grupos iniciales.

1. Enquesta de la Regió M etropolitana de Barcelona 1990. Condicions de vida i hàbits de la població. Barcelona: Institut d'Estudis M etropolitans (varios volúmenes). 


\subsubsection{El tercer nivel de análisis}

Sobre la base de los tipos iniciales y del conocimiento que de los mismos se ha obtenido en el segundo nivel, en la investigación se llega a un tercer nivel de análisis extrayendo de nuevo una segunda muestra cualificada en el interior de los tipos. La información ha sido obtenida ahora de entrevistas semidirigidas a los individuos escogidos. La base del guión semiestructurado se ha elaborado sobre tres líneas fundamentales. Primera, la centralidad de cada uno de los ámbitos (TP, TR, TL) en el presente de su vida diaria, días laborables, festivos, así como su articulación mutua. Segunda, la centralidad de cada uno de los ámbitos en el pasado - aspectos sociobiográficos- , el presente y el futuro- aspectos de proyecto relacionados con dichos ámbitos. Tercera, la articulación de las temporalidades para cada uno de los ámbitos. Se trata de identificar en cada uno de los tipos lo que hemos denominado «estructuras u orientaciones sentido o simbólicas vinculadas a los ámbitos». Los resultados tratan de sobreidentificar la identidad de cada uno de los tipos iniciales y dar cuenta, en su caso, o bien de la coherencia y consistencia de cada uno, o bien de su disgregación o inconsistencia en este nivel de análisis.

\subsection{O bjeto y objetivos de la investigación}

El aspecto más original de la investigación, y el objetivo fundamental, consiste en profundizar en las vinculaciones mutuas que se dan, en el fluir de la vida social, entre el ámbito del trabajo productivo,T P, el del trabajo reproductivo, $\mathrm{TR}$, y el del tiempo libre, TL. Esta articulación es precisamente - bien como relación de predominancia y dependencia de unos ámbitos sobre otros o bien como relación de equilibrio- el objeto específico de nuestro análisis, y no tanto el de cada uno de los ámbitos en sí. Precisamente porque nuestra vida social, individual y grupal, no está parcelada en sectores dispares y clausurados, este punto de vista y objetivo general adoptados nos parece no sólo pertinente sino también eficaz. Esta interacción entreámbitos y su correspondiente articulación constituirá la base o materia prima de lo que llamaremos «vida cotidiana».

La vinculación que pretendemos no se limita, como anunciábamos en la introducción, a la existente entre los ámbitos productivo, reproductivo y de disposición del tiempo libre en un nivel objetivable a partir de datos de la encuesta. Esta es una articulación que llamamos de «primer nivel» entre las tipologías iniciales de TP, TR y TL. La base fundamental de su contenido son las prácticas y atributos de los individuos objeto de estudio. Pero buscamos también analizar la interacción entre los diferentes niveles de nuestra actuación social. Así pues, aparece un segundo nivel que es el que trata de ver cómo se constituyen en cada uno de los ámbitos diferentes tipologías de las representaciones y actitudes y de éstas entre sí y, a partir de ella, en qué medida es coherente con la de los datos objetivables o atributos. En un tercer nivel se analiza lo que hemos denominado las «orientaciones o estructuras de sentido». Se trata tanto de los con- 
tenidos intencionales y proyectivos como de las referencias experimentales e históricas que dan sentido y coherencia a nuestra existencia social.

Pretendemos aś ir más allá de una visión puramente descriptiva y estática tra tando de llegar a aspectos más dinámicos, actitudinales y simbólicos de la vida cotidiana a partir de la comprensión de cómo y por qué se configuran unas u otras formas de vida cotidiana, fundamentalmente, primero, desde la elaboración y captación de los grupos sociales que están presentes en las diferentes tipologías y, segundo, examinando las coherencias y homologías que surjan entre los diferentes niveles, así como sus contradicciones.

\section{La aproximación multiestratégica de nuestra investigación}

Con esta visión general del proceso seguido en la investigación, haremos algunas reflexiones sobre temas o aspectos de la misma que la sitúan dentro de lo que hemos denominado «aproximación multiestratégica», no tanto con la idea de validar el procedimiento sino con la de contribuir a su concreción empírica y enriquecimiento.

\subsection{U na estrategia para la emergencia de la teoría y para la validación recíproca y parcial de aspectos parciales de la misma}

Como hemos adelantado, la aproximación realista o multiestratégica no se identifica ni con el paradigma de investigación que representan las teorías de rango medio, ni con el de las teorías de base. Tal y como se desprende del apartado anterior, en la investigación realizada no se pretende validar una teoría ad hoc, sino encontrar y generar conceptos o elementos en vistas a la elaboración de una teoría que dé cuenta de la interconexión entre diversos ámbitos sociales, dentro de un marco general de identificación y conceptualización de la vida cotidiana. Si solamente cumpliera esta condición podría ser contemplada claramente como teoría de base. Pero, por otro lado, algunos de los métodos y procedimientos utilizados, por exigencias mismas de lo investigado, son claramente cuantitativos, al menos en las dos primeras fases de la investigación.

Con relación al primer punto, búsqueda de elementos conceptuales en vistas a la generación de una teoría de articulación entre ámbitos sociales, hemos de decir que, aunque partimos de una concepción general de la vida cotidiana, tal concepción no tiene, en nuestra investigación, el rango de teoría en sentido estricto. Se trata más bien de unas líneas de contenido intuidas. ¿C uáles son estos apuntes de un esbozo teórico?

Primero, el paradigma de la vida cotidiana se entiende en la investigación, como el marco conceptual y teórico de una realidad social compleja que va más allá de la división que de los hechos sociales establecen las sociologías particulares o especializadas. Así, y concretamente en esta investigación, la vida cotidiana será considerada como una realidad social que corresponde a la articulación que se realiza entre el ámbito del trabajo, productor de bienes y 
servicios,T $\mathrm{P}$, el ámbito de las actividades que producen y reproducen la persona humana y las actividades de reproducción de uno mismo, TR, y, en fin, el ámbito de las actividades de tiempo libre,TL. Se entiende, además, que dicha articulación de ámbitos no se realiza sólo en el nivel factual y objetivable - actividades y prácticas-, sino también, y es uno de los elementos interesantes de nuestra investigación, en los niveles de las representaciones, actitudes y elementos simbólicos y estructuras de sentido.

Como puede observarse, nada presupone en tal modelo de vida cotidiana una teoría perfectamente construida e hilvanada disponible para ser validada. $M$ ás bien se trata de un contenido general como marco de referencia en el que se inscribe la investigación. No hay una formulación general adaptable a una validación a través de «tests de ajuste». M ás bien lo que pretendemos consiste en perfeccionar dicha concepción y, en todo caso, evaluarla interpretativamente en vistas a la posterior constitución de una teoría consistente. Con todo, esta reflexión no es óbice para que se den, en el interior de este marco interpretativo general, elaboraciones teóricas más perfiladas en al guno de los niveles y ámbitos que pueden ser validadas: concretamente en el ámbito del TP la teoría de la segmentación del empleo en el mercado laboral con rango de teoría media.

Segundo, con relación a la utilización de procedimientos cuantitativos en el proceso de la investigación, el primero y el segundo nivel han sido cubiertos con métodos y técnicas en la dirección cuantitativa, ya que los objetivos lo exigían y la información obtenida y requerida lo permitía. La constitución de los tipos de base (tipología de la segmentación laboral, T P; del trabajo reproductivo, TR, y de disponibilidad de tiempo libre, TL), provienen de los datos de una encuesta estandarizada con muestreo al eatorio y con contenidos tratados como variables. C ada una de las tipologías, a su vez, son cuantificables, lo que permite construir una tipología general de articulación por idénticos procedimentos (ver el artículo «La articulación de los ámbitos a partir de la base temporal», en este mismo volumen). Los procedimientos empleados en la construcción de estas tipologías son de tipo multivariable, en particular los de log-linear, análisis de componentes, de correspondencias y cluster.

\subsection{El mapa de investigación o los cortes analíticos y la vinculación entre ellos}

El resumen de los niveles y objetivos de la investigación presentada da ele mentos suficientes para comprender la naturaleza multinivel de la misma y la necesaria vinculación entre ellos. Ambas características, niveles y relación, son muy definitorias de la perspectiva multiestratégica: es el objeto mismo del contenido de la investigación quien marca la pauta y la necesidad del empleo de estategias diferenciadas y articuladas.

Concretamente en nuestra investigación la construcción del objeto, tomando como modelo genérico de análisis la idea de vida cotidiana, no se reduce sólo a las condiciones generales de vida o a las estructuras contextuales, ni tampoco exclusivamente a los proyectos, intenciones, principios de actuación 0 valores, ni únicamente a las representaciones y actitudes adoptados ante los 
hechos sociales. D e hecho, el objeto de estudio es un conglomerado articulado de todos estos elementos y factores, un producto de su interdependencia. El objeto no es tampoco un sólo ámbito, sea el del TP, el del TR o el del TL. Es, de nuevo, la articulación de ellos lo que nos interesa y ello en los diversos niveles que constituyen la personalidad social: actividades, representaciones, actitudes y construcciones simbólicas. Retomando la niveles de D . Layder, tendríamos en nuestra investigación las siguientes correspondencias.

El «sí mismo»: incluye las relaciones individuales con el entorno social concretado en la experiencia biográfica. C orresponde al tercer nivel de análisis que hemos denominado de las «estruturas de sentido» (entrevistas). Se trata de identificar los contenidos experimentales, intencionales y proyectos que dan coherencia a la vida social del actor social. Aunque la recogida de información sea individual, el objetivo consiste en enriquecer e interpretar los tipos originales a partir de la incorporación de la información con dicho contenido.

En la «actividad situada» se focalizan los aspectos dinámicos emergentes de la interacción social. Estenivel no ha sido considerado en esta investigación, ya que no hemos realizado ningún análisis de situaciones de interacción.

El «marco o composición», que comprende las formas intermedias 0 contextos de contenido: corresponde en nuestra investigación a las tipologías de base en cada uno de los ámbitos, TP, TR y TL, y sobre todo a la tipología de articulación entre ellas.

El «contexto» se refiere al marco general macrosocial que proporciona el más amplio entorno de la actividad social. Corresponde en nuestra investigación a la reconceptualización o recaracterización de las tipologías precedentes a partir de datos provenientes de indicadores sociales y estructurales, como categorías socioeconómicas o socioprofesionales, género, edad, etc. resultados de la encuesta metropolitana referida. Ambos, ámbitos y estructura (marco y contexto), partes integrantes del objeto construido, configuran el marco contextual en el que tomarán lugar, socialmente hablando, los otros dos niveles.

Al mismo tiempo, en la construcción de nuestro objeto de estudio se dan unos supuestos de vinculación entre dichos niveles introduciendo la idea de coherencia versus incoherencia entre ellos que supone para cada tipo la incorporación de la información del siguiente nivel. ¿L os contenidos de las representaciones 0 actitudes sobre los ámbitos tratados, TP, TR y TL, mantienen, disuelven 0 invierten la consistencia de los tipos construidos en el primer nivel? ¿ldem con su articulación? ¿Pasa lo mismo con los contenidos elaborados en el tercer nivel, es decir, con las construcciones simbólicas?

\subsection{La utilización de los métodos cuantitativos y cualitativos en el análisis}

En nuestra investigación ambos procedimientos tienen una función de consolidación y validación mutua con relación a la emergencia y constitución de una teoría. Se ha de insistir en el hecho de que la elección de ambos métodos, dentro de la misma investigación, no viene motivada por una posición metodológicamente ecléctica ni tan siquiera por el brillo y la elegancia que puede dar 
un barniz de complementariedad, sino que está basada en los cortes y planos que se consideran en el objeto analizado y sobre todo en las exigencias que emite de su necesaria vinculación.

Concretamente, la construcción de las tipologías, tanto en cada ámbito como la articulación de las mismas en el primer y segundo nivel de análisis, es exclusivamente cuantitativa. I dénticamente lo es el modo de recogida de información y el análisis primario de los resultados de la encuesta inicial. Pero el tercer nivel de análisis se ha realizado por medio de entrevistas semidirigidas y con procedimientos de análisis de contenido temático cualitativo según una parrilla previa que daba al mismo tiempo las orientaciones interpretativas. Además, la lógica del proceso conduce a una mutua coval idación de resultados y métodos, ya que se trata de ir construyendo sucesiva y acumulativamente la identidad de cada tipo.

\subsection{La tipología estructural y la tipología comparativa}

Uno de los procedimientos de análisis más afín a la aproximación multiestratégica es, para D. Layder, el método tipológico. N o es de extrañar que sea una línea de investigación en la que los objetos construidos no tienen la «categoría» de teoría como expresión formal de relaciones expresables analíticamente entre sus conceptos o variables como por ejemplo las causales. Precisamente, en los al bores de la génesis de una teoría, las construcciones tipológicas pueden ser la expresión metodológica más pertinente, en particular si pretendemos esbozar, precisar y relacionar conceptos más allá de sus indicadores directos. El análisis tipológico encaja bien con un propósito que va más allá de una simple descripción clasificatoria e incluso taxonómica para adentrarse en la elaboración de una teoría a partir de la interacción y articulación de dimensiones y en la localización de los tipos en ellas. En nuestra investigación los conceptos primeros son más o menos balbucientes y se realizan y concretizan en cada uno de los ámbitos por la tipología construida en ellos a partir de determinados indicadores/dimensiones. D el conjunto surge una nueva tipología y con ella una incipiente teoría y la explicitación de un nuevo concepto, el de la articulación de los tres ámbitos originando tipos de vida cotidiana.

La tipología construida, como la de los ámbitos de partida, no sólo tiene interés por su carácter de articulación vinculando ámbitos distintos entre sí, sino que también lo tiene por ser de gran potencial comparativo. Ambas características son específicas y propias de la aproximación multiestratégica: estructural en cuanto al proceso de construcción y en cuanto que, simultáneamente, articula elementos de distintos niveles; comparativa porque esta tipología es la base para poder contrastar y cotejar, entre tipos diferentes, otras características, como son actitudes, representaciones y orientaciones de sentido.

\subsection{M uestra estadística, muestra teórica y muestra ecológica}

La función corriente del muestreo al eatorio es la de asegurar la representación de toda la población salvo el riesgo de un error calculable. Para establecer dicho 
error y el correspondiente número de muestra se estiman los valores de al guna variable poblacional. En la muestra estratificada se introducen elementos que hacen que, además de mantener la imprescindible finalidad de representación, permite también tipificar la población con relación a las variables/criterios, que serán tanto más eficaces cuanto más se acerquen a las variables estructurales de la población considerada. Q uiere esto decir que si se utilizan en la construcción de la muestra variables que tienen una función explicativa importante en el modelo de análisis, la muestra, además de ser más precisa y eficaz, contendrá elementos teóricos que nos permitan el control comparativo más ajustado entre tipos.

Concretamente, en la investigación comentada se dan tres muestreos sucesivos.

El primer muestreo es el de la encuesta de base para la construcción tipológica inicial. Se trataba de un muestreo estratificado, por lo que posee, aunque sea al eatorio, un carácter parcialmente orientado por la teoría, en el sentido que las variables criterio utilizadas para construir los estratos fueron elegidas según los objetivos de la encuesta. Esta muestra fue realizada, incluso en la primera encuesta, por algunos de los miembros del equipo que han participado en la investigación presentada (C. Lozares y P. López, 1991). Por consiguiente, se asegura una continuidad y comprensión metodológica en el trasvase de los datos de partida para las siguientes fases. D e la encuesta correspondiente se extraen los datos para construir las tipologías de cada ámbito y la de articulación entre ellos. Se ha de insistir, pues, en que los tipos construidos en la primera fase de la investigación son representativos del conjunto de la población de la Región M etropolitana de Barcelona.

$\mathrm{D}$ ados el contenido y la extensión de los datos que sirven para su construcción, la tipología de articulación entre ámbitos (y los tipos correspondientes) se puede decir que:

1) es representativa de una gran población y, por consiguiente, posee un elevado grado de generalidad en sus resultados, como lo tendrá para los sucesivos análisis;

2) tiene un carácter eminentemente denotativo y objetivable; es, en este sentido, el resultado de la simplificación y síntesis de un largo proceso de análisis de una gran encuesta desde la perspectiva que nos interesa. Participa, pues, de la consistencia y coherencia que estos análisis extensos y generales acostumbran a tener. Por ello es un punto de inicial identidad, referencia y contraste para las tipologías que puedan surgir en los restantes niveles: representaciones, actitudes y construcciones simbólicas;

3) es la base muestral para la segunda fase y para la tercera; es decir, a partir de los resultados obtenidos en este nivel se puede conseguir una «especie» de estratificación muestral en la que tienen predominancia decisiva los aspectos de contenido. En este sentido, la primera fase o el análisis del primer nivel tiene no sólo una función estratégica de contrastación y compa ración, sino también instrumental. Es un aspecto metodológico importante que habrá que profundizar. 
El segundo muestreo se hace a partir y sobre los tipos hallados en el primer nivel de análisis. Acentúa más el carácter cualificado (cualitativo) y podemos denominarla «muestra tipificada» y también «teórica y ecológica». Por consiguiente, para nuestros propósitos y conceptos manejados, el universo estudiado se configura y exclusiviza en y por dichos tipos. Son, en consecuencia, tipos de articulación de vida cotidiana al nivel de los indicadores elegidos y manejados en la primera fase. El interés de la segunda fase está precisamente en tomar estas entidades como sujetos de investigación. Se estudian las actitudes y representaciones con relación a nuestro objeto de estudio así como los modos de vida. Se han realizado dos centenares de entrevistas distribuidas convenientemente según el número de población de y en cada uno de los tipos. Por consiguiente, no se pretende que las consecuencias de esta segunda fase sean representativas de toda la población de la región en el sentido estadístico de la palabra, sino que se trata de una aproximación suficientememente cualificada para la identificación de los tipos en las actitudes y representaciones. Como la variabilidad en el interior de los tipos con relación a las variables de base que los constituyen es reducida (desviación reducida), éstos adquieren una consistencia suficiente para que cada individuo de un tipo pueda ser supuesto y considerado como semejante al conjunto de los que lo forman y, por consiguiente, represente e identifique al tipo. Esta segunda muestra es el instrumento para sobreidentificar a los tipos a partir de los datos que son propios de este segundo nivel, representaciones y actitudes y modos de vida. Se trata de una muestra teórica, pues al estar realizada sobre los tipos precedentes supone una teoría, al menos en los resultados obtenidos, acerca de la vinculación de ámbitos o modos de vida cotidiana. Se habla de una muestra ecológica porque, aunque de carácter cualitativo, indica no sólo criterios para el egir a los individuos, sino que los tipos de los que se extraen son verdaderos contextos estructural es de las variables de los ámbitos. Los individuos de cada tipo a los que se realizan las entrevistas y nuevas intervenciones están socialmente bien contorneados y ecologizados social mente.

El tercer muestreo se ha realizado sobre la base de los resultados de la segunda fase una vez identificados los tipos con las características añadidas a partir de las representaciones y actitudes. Este tipo de muestreo no es cualitativamente diferente del precedente, solamente se acentúan aún más su cualificación y su carácter teórico en el sentido atribuido precedentemente. D ado que se trata de entrevistas semidirigidas, el número elegido ha sido considerablemente menor.

0 tra consideración importante es que precisamente este muestreo sucesivo sobre la base de los resultados de los niveles precedentes es lo que nos permite vincular niveles de análisis dentro de mapa del objeto construido que de otra manera hubiera sido imposible realizar.

\subsection{Reflexión final}

Retomando la idea inicial, recordemos que no pretendíamos en estas páginas ni fundamentar epistemológicamente la aproximación multiestratégica, ni vali- 
darla a partir de la exposición de un caso. El propósito consistía solamente en corroborar y abundar en una aproximación que, además de ser frecuente en la práctica de la investigación sociológica, es real ista y eficaz en sus resultados y, sobre todo, está más próxima a los hechos analizados. Por lo demás, reflexiones en este sentido pueden contribuir a la constitución de un cuerpo extenso de experiencias de investigación que van sentando las bases a una teorización específicamente epistemológica en torno a la aproximación multiestratégica.

D iversas y serias cuestiones se nos plantean en esta línea de tratamiento que tienen que ver más con la manera de resolver, si es posible, determinados dile mas profundos que atraviesan la sociología y su metodología que con encontrar soluciones pragmáticas a determinados problemas de método y técnicas que surgen de la aplicación de esta aproximación metodológica. Somos de la opinión que el avance en este tipo de tratamientos será correlativo a un progreso paralelo en la resolución de estos dilemas. ¿C uáles son éstos? Son de sobra conocidos, sirvan como enumeración: actor/(inter)acción versus estructura; aproximación macro versus aproximación micro; individualismo versus holismo; determinación versus indeterminación; redes versus atributos; acción instrumental versus no racional, etc. Se puede seguir, pero este listado ya muestra los principios o dimensiones que clasifican profundamente los diversos métodos y metodologías de aproximación al objeto investigado. Evidentemente no se puede esperar a su resolución para continuar con la práctica investigadora, por ejemplo, en una dirección multiestratégica que trata de resolver estas oposiciones (y quizás contradicciones o dilemas irreconciliables) por una vía pragmática dado que los objetos construidos lo reclaman. H e ahí un buen campo para los filósofos de la ciencia. Para nosotros queda considerar si estos métodos nos aportan más información sobre la realidad social con la condición de que no esté sesgada y no se rompan al gunos esquemas lógicos, entre otras muchas vigilancias el ementales. Este avance contribuirá a un mayor «acercamiento» entre los polos de dichos dilemas.

\section{Bibliografía}

BECKER, H . (1970). Sociological Work. Chicago: Aldine.

D O U GLAS (1976). Investigative Social Research. Beverley H ill: Sage.

Glaser, B.; Strauss, A. (1967). TheD iscovery of Grounded Theory. Chicago: Aldine. GoLDTH ORPE, J. (1980). Social M obility and Class Structure in M odern Britain. Oxford:

Clarendon.

IBÁÑ EZ, J. (1979). M ás allá de la Sociología. M adrid: Siglo XXI.

- (1986). «Perspectivas de la investigación social». En García FerRando, M . y otros.

El análisis dela realidad social. M adrid: Alianza U niversidad.

LAYD ER, D. (1993). N ew Strategies in Social Research. Cambridge, M A: Polity Press.

LOFLAN D, L. (1971). «n the Presence of Strangers: A Study of Behavior in Public

Setting». Working Paper, 19. M ichigan: C entre for Research on Social O rganizations, University of M ichigan.

LOZARES, C. (1991). «La complementarietat quantitativa i qualitativa: un cas d'analisi del medi popular a Suïssa», p. 17-25; «La complementarietat quantitativa i qualitativa: 
un cas d'analisi dels temporers de G inebra», p. 27-36. Tècniques qualitatives en Ciències Socials. Barcelona: Societat C atalana de Sociologia.

LOZARES, C.; LÓ PEZ, P. (1991). «El muestreo estratificado por análisis multivariado». En El pluralismo metodológico en la investigación social: ensayos típicos. G ranada: Universidad de Granada.

LOZARES, C. y otros (1988). Portrait des salariés romands. Lausanne: Editions d'en bas. RitZer, G. (1992). Contemporary Sociological Theory. N ueva York: M cGraw-H ill. St rauss, A. (1987). Q ualitative Analysis for Social Scientists. Cambridge: Cambridge University Press.

W RIGHT, E. (1980). «Class and occupation». Theory and Society, 9, p. 177-214. 